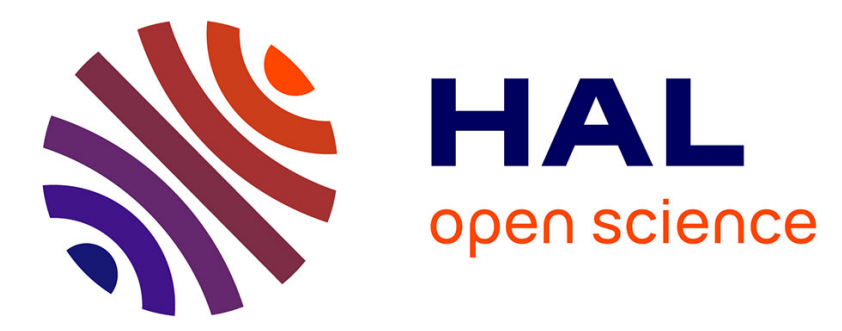

\title{
A Word Sense Disambiguation Method for Feature Level Sentiment Analysis
}

\author{
Umar Farooq, Tej Prasad Dhamala, Antoine Nongaillard, Yacine Ouzrout, \\ Muhammad Abdul Qadir
}

\section{- To cite this version:}

Umar Farooq, Tej Prasad Dhamala, Antoine Nongaillard, Yacine Ouzrout, Muhammad Abdul Qadir. A Word Sense Disambiguation Method for Feature Level Sentiment Analysis. 9th IEEE International Conference on Software, Knowledge, Information Management \& Applications (SKIMA 2015), Dec 2015, Kathmandu, Nepal. hal-01467595

\section{HAL Id: hal-01467595 \\ https://hal.inria.fr/hal-01467595}

Submitted on 31 Oct 2018

HAL is a multi-disciplinary open access archive for the deposit and dissemination of scientific research documents, whether they are published or not. The documents may come from teaching and research institutions in France or abroad, or from public or private research centers.
L'archive ouverte pluridisciplinaire HAL, est destinée au dépôt et à la diffusion de documents scientifiques de niveau recherche, publiés ou non, émanant des établissements d'enseignement et de recherche français ou étrangers, des laboratoires publics ou privés. 


\title{
A Word Sense Disambiguation Method for Feature Level Sentiment Analysis
}

\author{
Umar Farooq*, Tej Prasad Dhamala $^{\dagger}$, Antoine Nongaillard ${ }^{\ddagger}$, Yacine Ouzrout ${ }^{\S}$ and Muhammad Abdul Qadir ${ }^{\llbracket}$ \\ $* \dagger \S$ DISP Laboratory, University Lumiere Lyon 2, Lyon, France \\ Email: * Umar.Farooq@univ-lyon2.fr, † tejp_dhamala@live.com \\ $\S$ yacine.ouzrout@univ-lyon2.fr \\ ${ }^{\ddagger}$ CRIStAL Laboratory, CNRS UMR 9189, Lille University, Lille, 59650, France \\ Email: antoine.nongaillard@univ-lille1.fr \\ ${ }^{*}$ Computer Science Department, Abdul Wali Khan University Mardan, Pakistan

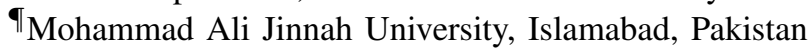 \\ Email: aqadir@jinnah.edu.pk
}

\begin{abstract}
Sentiment analysis is an automatic method used to determine that the opinion of a person about a subject is positive or negative. One of the most important tasks in sentiment analysis is to disambiguate the sense of words according to context. Most errors in sentiment analysis are because of improper sense disambiguation. Few methods for this purpose have been proposed in literature. However, they are not able to properly determine the context of word in a sentence. In addition, the lexicon dictionaries used by these methods lack word senses and also do not provide a context matching technique. These issues need to be addressed in order to improve the performance of sentiment analysis so that it can be used by customers and manufacturers for decision making. In this paper, we propose a feature level sentiment analysis system, which produces a summary of opinions about product features. A word sense disambiguation method is introduced which accurately determines the sense of a word within a context while determining the polarity. In addition, a heuristic based method is proposed in order to determine the text where opinion about a product feature is expressed. The results show that the proposed methods achieve better accuracy than existing methods.
\end{abstract}

Keywords-Sentiment Analysis; Contextual Polarity; Word Sense Disambiguation

\section{INTRODUCTION}

Sentiment analysis also known as opinion mining refers to the process of determining opinions or emotions expressed in text about a subject. Sentiment analysis is a wide research area at the intersection of different domains: natural language processing, computational linguistics and text mining. Although sentiment analysis is a recent research area, which has been introduced in 2001 [1], [2]. It arouses a lot of interest and numerous standalone applications are developed to identify sentiment and opinions, e.g., in product reviews, news, twitters and blogs [3]-[7]. Several sentiment analysis systems have been proposed in literature in order to classify the polarity in a document, sentence, phrase or about a product feature. In this paper we focus on feature level sentiment analysis where we determine that the opinion about a product feature is either positive or negative. The results of feature level sentiment analysis are very useful for both customers and manufacturers in order to make decisions. The customers can used the results in order to compare products based on different features to make purchase decision. Similarly, manufactures can use the results of feature level sentiment analysis to know the strengths and weaknesses of the product in order to improve it and to launch different marketing strategies.

One of the issues in sentiment analysis is word sense disambiguation. Word sense disambiguation is an automatic way to determine sense of the word within a context. Generally, word sense disambiguation is used to identify the intended meaning of a word in a sentence. However, in sentiment analysis it refers to the process of determining the sentiment orientation sense (i.e. positive, negative and neutral sense) of a word in a sentence within a context. Therefore, in sentiment analysis, words have three different senses, i.e. positive, negative or neutral. Similarly, the context of a word refers to other words around on which the sense of this word depends. Indeed, a word polarity may vary according to its context. As illustrated in Example 1, the word 'small' appears in both positive and negative senses depending on context. In hotel domain 'small' expresses negative opinion, however the same word expresses positive opinion in mobile and laptop domains. Countless descriptive words face similar situations like large, short, lightweight, long, removed, cheap etc. Most errors in sentiment analysis are due to the word appears in sentence in one sense but the polarity obtains from lexicon is of another [8]. In short, word sense disambiguation is very important to accurately determine a sentence polarity.

\section{Example 1:}

This mobile is small enough that it comes easily in my jeans packet.

The hotel is very small.

I have very small laptop which I can carry easily.

Most of the sentiment analysis systems determine the polarity without any sense disambiguation. Few authors proposed sense disambiguation methods [9], [10], however determining the context in a sentence and identifying it exact match in lexicon do not provide good results. Most of the sentiment analysis systems are based on pre determined lexicon dictionary of opinionated words. However, most of these lexicon dictionaries contain only the words with their associated polarities, rather than the words polarities with different senses [8]. Some 
lexicon building methods are proposed which obtained senses of words from WordNet. However, these lexicons do not support word sense disambiguation and also lack word senses. In addition, there are several words such as intensifiers and reducers which increases or decreases the polarity of other words. Such words include very, little, enough, deeply, entirely etc. These words can only be handled if the relationship between intensifier/reducer and modified word are identified accurately and if the polarity of modified word are correctly disambiguated. However, most of the existing methods are not able to handle properly such kind of words.

On the other hand, an issue concerned with feature level sentiment analysis is to determine the exact boundaries of the text where opinion about a product feature is expressed. In literature, three different methods, i.e. clause based [6], sentence based [11], [12] and adjective based [4], [13] methods are used for this purpose. The clause based method only considers the clause in which the product feature appears even if the next clause is also about the same feature. The sentence based method considers the whole sentence where a feature appears. However, when two or more than two features appear, this method considers the complete sentence for both/multiple features, although the opinion about one feature can be opposite to another. The adjective based method considers only the adjective used with product feature to determine the polarity while ignoring other opinion bearing parts of speech such as verb, adverb and noun. All these methods either ignore some portion of the text about a feature or consider irrelevant text for a feature while determining the polarity. If a sentiment analysis method is not able to accurately locate the exact text about a feature then how we can trust on the classification results.

Considering these issues, in this paper we propose a feature level sentiment analysis method, which extracts product reviews from several web sources and produces a summary of opinions about different features of the product. A heuristic based feature scope identification method is proposed in order to determine the exact text where opinion about a feature is expressed. In addition, a word sense disambiguation method is proposed which first builds a lexicon dictionary of words senses for a particular domain and then locates the context of a word in a sentence and obtains polarity from the lexicon accordingly.

This paper is organized as follows. In Section II, related works are presented and analyzed. Section III explains the proposed word sense disambiguation and feature scope identification methods. Finally, Section IV evaluates the performance of the proposed methods.

\section{RELATED WORK}

The majority of sentiment analysis systems determine the polarity without any sense disambiguation. Although word sense disambiguation is one of the most important tasks in sentiment analysis, still few methods are proposed in literature for this purpose. Plenty of errors in sentiment analysis are because of improper sense disambiguation [9] , i.e. a word appears in one sense however the polarity obtained from the lexicon is of another sense.

Some researchers such as Kim and Hovy [14], Yu and Hatzivassiloglou [15], Hu and Liu [12] and Grefenstette et al.
[16] begin by first creating lexicon dictionaries where words are associated with the prior polarity out of context. However. the contextual polarity of a word appeared in a phrase may be different than the word's prior polarity because a word may appears in different senses [9]. In addition, there are several words which prior polarity is difficult to define because they do not carry specific polarity by itself, such words include long, short, small, feel, think, deeply, entirely, practically etc.

In [9] a method to determine the contextual polarity of phrase is proposed. This approach is based on the fact that neutral clauses are frequent, therefore polar clauses are identified first. The context of word (which is a bag of three words: the previous word, the word itself, and the next word) is used with linguistic features such as modification features, sentence feature and structure features to determine the polarity of polar clauses. However, in this method the word context is used to determine the effect of enhancers, modifiers and negation instead of disambiguating the word sense. The MPQA (Muti perspective Question Answering Opinion Corpus) is used which list down only the words and phrases with their associated polarities rather than words with different senses.

A word sense disambiguation method at sentence level is proposed in [10] which is based on parts of speech pattern matching. While determining the polarity, parts of speech pattern of the sentence is extracted and compared with the WordNet glossaries in order to identify the appropriate sense in SentiWordNet. However, the parts of speech pattern matching do not achieve satisfying results because a word used in the same parts of speech pattern may not have the same sense. For example, in sentences: "the mobile is small" and "the hotel is small" the word "small" appeared in the same parts of speech pattern. However, in first sentence "small" is used in positive sense while in second sentence the same word is used in negative sense. This method also depends on SentiWordNet lexicon dictionary which have several issues as discussed later in this Section.

Most of the sentiment analysis systems are based on prebuilt lexicon dictionaries of opinionated words. Several dictionaries such as SentiSense [17], SentiWordNet [18], Micro-WNOp [19] and WordNet-Affect [20] are built which used WordNet information in order to automatically annotate the synsets with polarity scores. The issue in these lexicon dictionaries is that they list down only the common senses of a words which is based on WordNet. Indeed, the senses in WordNet are according to intended meaning and it is difficult to transformed them into sentiment orientation sense. In addition, these lexicon dictionaries do not support any sense matching mechanism in order to disambiguate the word sense. Some authors used the WordNet glossary for this purpose [10], however it does not achieve accurate results.

A word sense disambiguation method will only be effective if the following conditions are satisfied.

- An appropriate method is required to determine the context of word in a sentence.

- The context of a word not only depends on the words around but also on the topic discussed which may not be explicit. Therefore, it is also necessary to identify the topic discussed and its boundaries within the text. 
- $\quad$ The lexicon dictionary must have all senses of words according to contexts with their associated polarities instead of only listing words with polarities.

- The senses must be according to sentiment orientations context instead of based on intended meaning (as extracted from WordNet in most existing lexicon dictionaries).

- An appropriate context matching method method is required, which the lexicon dictionary must support in order to determine the appropriate context in the lexicon so that the polarity (sense) of a context can be obtained.

Indeed, it is very difficult and time consuming to obtain all senses of words manually for a domain which will be repeated again for another domain [21]. Similarly, it is also challengeable task to automatically extract all words used in different contexts in distinct domains. However, it can be possible to extract words with different contexts automatically only for a particular domain. Therefore, in this research work we propose a mechanism which obtains all the senses of words in a particular domain and also satisfies the above conditions.

\section{WORD SENSE DiSAMBIGUATION}

In sentiment analysis it is very important to disambiguate the context of the word in order to improve the accuracy. A word may appear in positive, negative or neutral senses if used in different application domains. Even in the same domain a word may appear in different senses if used with different product features. As illustrated in Example 2, the word 'long' used in mobile domain, expresses different opinions (i.e. positive and negative) when used with different features (i.e. battery and application). A sentiment analysis method which is not capable to disambiguate the sense will classify these sentences wrongly.

Example 2:

The battery works for a long time.

The application takes long time to load.

The existing word sense disambiguation methods have two main problems. 1) These methods are not able to properly determine the context of word in a sentence. 2) In addition, the lexicon dictionaries (on which the methods depend) do not support the sense disambiguation because of two reasons. First, these lexicon dictionaries either lack word senses or only contain words with their associated polarities rather than the words polarities with different senses [8]. Secondly, they do not provide a context matching method in order to obtain the polarity of the accurate sense from the lexicon. Based on these issues, we propose a method which determines the context of word in a sentence and builds a lexicon dictionary which contains most of words senses in a particular domain. In addition, this method also provides a context matching mechanism in order to obtain the polarity of the corresponding context from the lexicon.

The context of word in a sentence depends on four things, i.e. global topic, local topic, global context and local context. We need to disambiguate both global and local context. The global context is the context of a word in a specific domain of application, for example, electronics, sport goods and automobiles etc. Similarly, the Local context is the context of a word within a sentence which depends on the words around. Therefore, the proposed method for word sense disambiguation is divided into two steps (i.e. Building Lexicon Dictionary and Local Context Disambiguation). The global context is disambiguated by developing a lexicon dictionary only for a specific application domain. In addition, the local context is disambiguated by considering local topic discussed, linguistic characteristics, and the semantic relationships between different parts of speech within the sentence.

\section{A. Building Lexicon Dictionary}

Word sense disambiguation relies on lexicon dictionary of words senses. If a lexicon dictionary either lacks word senses or only contains a list of words instead of senses, then word sense disambiguation method will not be effective. We propose a method to identify the senses of words in a specific domain to build a lexicon dictionary. The following steps are followed to build sense-tagged lexicon. Reviews about a specific product are extracted from different review sites. Product features are extracted from the reviews using existing feature extraction method [4]. The text where opinions are expressed about different features are located in reviews. All opinionated words used to express opinions about features are extracted with the contexts in order to obtain seed set of word senses. The polarities of the seed of word senses are annotated and expanded by obtaining synonyms and antonyms from WordNet. All these steps are explained in the remaining part of this section.

1) Product Reviews Extraction: In order to build a lexicon dictionary for specific domain, the reviews about a product are extracted from Web. For this purpose, a wrapper is developed which extracts reviews automatically from e-commerce and review sites such as Ebay, Cnet and Amazon. The wrapper is fully automatic, the user simply need to select a product and the wrapper automatically locates the web pages (containing reviews about that product) and extract reviews from all those web pages.

2) Product Feature Extraction: In this step the proposed method in [4] is implemented in order to determine the product features. The users are also allowed to modify the results in order to improve the accuracy. The users can select only the most frequent features, map features identified by the system as separate features which are actually single feature, however different names are used (e.g. battery life and battery, application, program and app etc.) and even can define their own features. However, for building lexicon dictionary we considered all the features about which the customers expressed opinions.

3) Feature Scope Identification: The chunk of text where opinion about a feature is expressed is called the feature scope. The feature scope can be a single clause, multiple clauses or even the whole sentence. Feature scope identification is very important because sense disambiguation not only depends on the topic discussed (i.e. product feature) but also on the boundaries of text where this topic is discussed. In fact, more than one features can be discussed in a single sentence. Similarly, 
a feature can also be discussed in multiple clauses. It is very important not only to separate opinions about one product feature from another but also to identify all those consecutive clauses where opinion about a single feature is expressed. In literature, three different methods, i.e. clause based [6], sentence based [11], [12] and adjective based [4], [13] are used to identify the scope of feature. However, all these methods are not able to identify the feature scope correctly. We propose a heuristics based feature scope identification method which gives more accurate results. This method uses the following heuristics rules which are based on: the clause in which the feature appears, parts of speech pattern, noun clause, conjunction used, and the reference words used at the beginning of succeeding clause.

- The scope of a product feature starts from the clause where a feature appears and it may or may not be extended to the succeeding clauses.

- If the succeeding clause does not contain a noun (i.e. not a noun clause) then the scope of the feature is extended to the next clause because no other topic or feature is discussed.

- However, if the succeeding clause contains another product feature then it means that the scope of feature discussed in preceding clause is limited only to that clause.

- On the other hand, if the succeeding clause is a noun clause but the noun is not a product feature, then we have three choices. i) If the conjunction used is either 'AND' or 'OR' and the same parts of speech pattern up to three words is followed on either sides of conjunction then it is most likely that the same feature is also discussed in the next clause. ii) If there is a reference word at the beginning of the succeeding clause then there is highly likelihood that the scope is extended to the next clause even if it is a noun clause. Reference words (e.g. it, this, that, these, those, which, such etc.) are used to refer back to the object or idea that have been mentioned earlier. iii) If both cases i) and ii) do not exist, then it is highly likelihood that the scope of the feature is only limited to the clause in which it appeared.

The algorithm 1 is proposed to identify the feature scope which is based on the heuristics rules. The inputs to the algorithm are the Product features set $P F$, the reviews set $R$, the reference words set $R W$ and conjunctions set $C$. The output of the algorithm is the feature scope $F S$. First, the clause $F C L$ in which the feature appeared is identified which is assign to the candidate feature scope $C F S$ (Line 1-2). The remaining steps identify that whether the feature scope $F S$ only contains the $C F S$ or extended to the succeeding clause $S C L$. If the $S C L$ is not a noun clause $N C L$ then both $C F S$ and $S C L$ are included into the feature scope (L 3-5). If the succeeding clause is $N C L$ and the noun $N N$ which appeared in this clause belongs to the feature set $P F$, then only $C F S$ becomes the $F C$ (L 6-8). If the $S C L$ is $N C L$, the conjunction $C$ used is neither 'AND' nor 'OR' and $S C L$ does not have reference word at the beginning, then $F S$ is limited only to $C F S$ (L 9-11). Similarly, if the $S C L$ is $N C L$ and either the conjunction used is OR/AND or $S C L$ contains $R W$ at the beginning then both $C F S$ and $S C L$ are included into the $F S$ (L 12-14). If there is another $S C L$ then all steps from 3-14 are repeated in order to check that weather this succeeding clause is also included in the $F S$ or not. In this way, the algorithm identifies the individual clause, multiple clauses or even the whole sentence where opinion about a product feature is expressed.

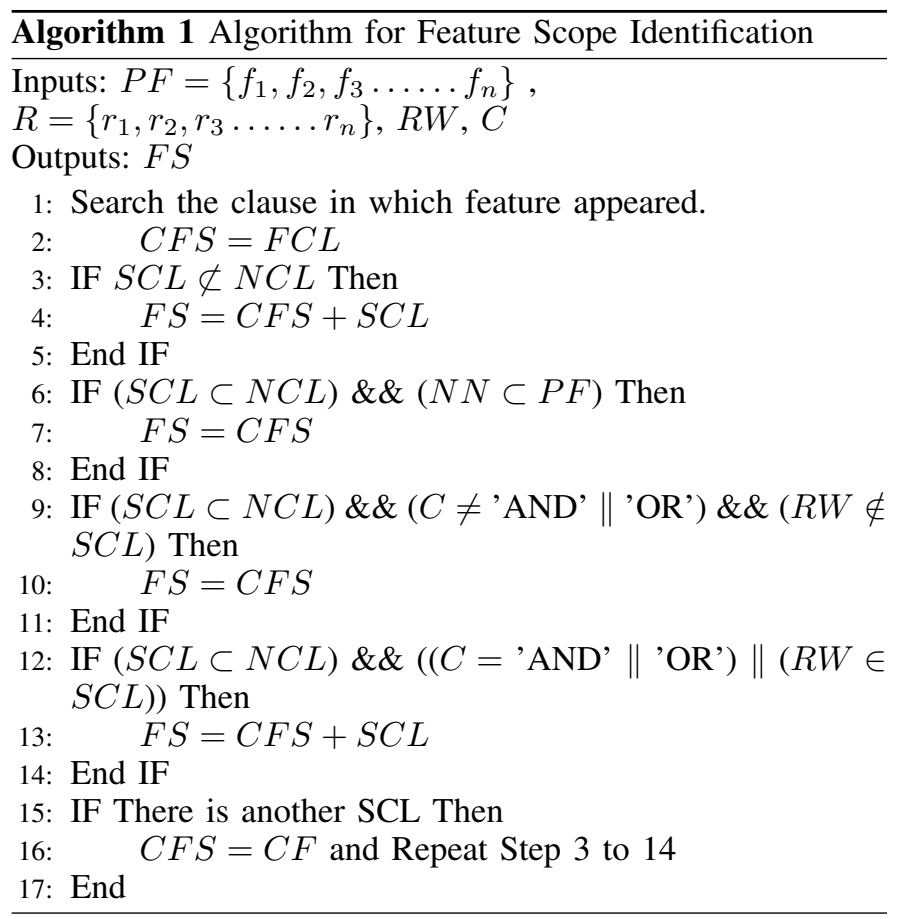

4) Building Seed Set of Word Senses: In order to build seed of word senses, feature scopes identified for all product features in the previous step are considered to automatically extract words in different contexts. Only the opinionated parts of speech which carry opinions (i.e. adjectives, verbs, adverbs and nouns) are extracted with their contexts. As the context of a word depends on other words around with which the word has some semantic relationship. Therefore, for each opinionated part of speech we proposed a semantic relationship in order to identify the context. The following four semantic relationships i.e. for adjective JJ, verb VB, adverb RB and nouns NN are used to identify the context and to extract these words with their contexts automatically.

1) The most important part of speech is adjective which describes a noun. This noun is usually the product feature or some other noun which further describe the product feature PF. This means that the polarity of an adjective depends on the product feature which it describe (i.e. JJ, NN/PF).

2) The verb describes some action related to product feature and hence the polarity of verb can be determine while considering the product feature in mind (i.e. VB, NN/PF).

3) Similarly, the adverb describes or modifies either an adjective or a verb which further describes a noun or product feature. The adverb do not have a direct relationship with product feature and hence the effect or polarity of adverb can be determine by considering 
the whole sequence of relationship (i.e. $\mathrm{RB}, \mathrm{JJ} / \mathrm{VB}$, NN/PF).

4) Sometime the noun (other than the product feature) which carries opinion also describes some aspect of the product feature (i.e. NN, PF).

The polarities of words in different contexts in seed set are annotated by a human annotator. For this purpose a user interface is built where each word is assign either positive, negative or neutral polarity according to the context, within the rang of 0 to 1 , based on the strength of positive or negative opinion expressed. Manual annotation is used because the automatic annotation methods are not able to accurately annotate the words within a context. In addition, one single incorrect annotation in the seed set can affect large quantities of synonyms and antonyms in the target lexicon in seed expansion.

5) Seed Expansion: In order to build lexicon dictionary, the seed set is further expanded automatically by obtaining the synonyms and antonyms from WordNet. The synonyms set obtained for a word used in a specific context is given the same polarity as that word has. On the other hand, the antonyms are assign the opposite polarity of the word for which they are obtained. For example, if a word appeared in single context and five synonyms and antonyms are obtained, then all synonyms in the same context are assigned the same polarity and antonyms within in the same context are assign with opposite polarity. On the other hand, if there is a word in three different contexts and five synonyms and antonyms are obtained, then by using this method thirty other words with these three senses are obtained (i.e. $5^{*} 3=15$ by synonyms set and $5^{*} 3=15$ for antonyms set). In this way, the seed set of word senses is expanded in order to build the lexicon dictionary. The lexicon dictionary contains the opinionated words with different contexts (i.e. semantic relationships) as well as the senses (i.e. polarities).

\section{B. Local Context Disambiguation}

This section explains that how the local context of a word within a sentence or feature scope $F S$ is disambiguated while determining the polarity. Before disambiguating the local context first the product features and feature scopes are identified using the corresponding methods. While determining the polarity of a $F S$ or sentence, first the context of every opinionated word are determine using the four semantic relationships proposed in section III-A4. Second, the word with their context is searched in the lexicon and the polarity of the match context is obtained from the lexicon dictionary. If exact match is not found in the lexicon then the polarity of the partial match context is obtained. Lets we want to find the polarity of sentences in Example 3. In first sentence, only one opinionated part of speech that is bright/jj is used to express opinion. In second sentence, three opinionated parts of speech, i.e. works/VB, long/JJ and time/NN are used to express opinion about battery $P F$. First, the contexts of these words are determined using the following three semantic relationships.

1) The JJ 'bright' describes an aspect of feature (i.e. screen/PF bright/jj+Screen/PF).

2) The VB 'work' describes an action related to feature battery/PF (i.e. work/VB+battery/PF).
3) The JJ 'long' describes the NN 'time' which further describes an aspect of battery PF (i.e. long/JJ+time/NN+battery/PF).

In second step, these words are searched according to these contexts (i.e. bright/jj+Screen/PF, work/VB+battery/PF and long/JJ+time/NN+battery/PF) in the lexicon. The polarities of the match contexts are obtain from the lexicon in order to determine the polarity of the whole sentence.

Example 3

The screen is bright.

The battery works for a long time.

It is easy to identify the semantic relationships between parts of speech when each part of speech appears only once in a sentence. Conversely, it is challengeable task to identify the relationships when each part of speech appears multiple times in a single sentence or even in a single clause. On the other hand, it becomes more difficult if multi topics discussed in a single sentence. Therefore, in order to make our task easier we already make sure that the feature scope is only about one topic (i.e. about a product feature) using feature scope identification method. We used the following heuristic rules to automatically determine the semantic relationships between opinionated parts of speech.

- Usually, parts of speech have relationships within a clause. Only in the following cases the relationship is extended to the succeeding clause. a) When there is no noun in succeeding clause then the opinionated parts of speech have relationships with feature discussed in preceding clause. b) Similarly, when there is a noun but the noun is not product feature the opinionated words in succeeding clause have relationships with the words in preceding clause.

- Adverb is usually used before an adjective or a verb, which it modifies.

- The adjective and verb have relationship with nearby noun (which may be product feature) within a clause. If there is no noun in the clause then the adjective and verb will have relationship with the noun discussed in preceding clause.

\section{RESULTS}

We performed three sets of experiments in order to evaluate the performance of our proposed methods. The first set of experiments is performed to measure the performance of feature scope identification method. The second set of experiments is performed to evaluate local context disambiguation method. The last set of experiments is performed to evaluate the performance of overall feature level sentiment analysis method. As, the word sense disambiguation method depends on both feature scope identification and local context disambiguation, thus both first and second set of experiments collectively evaluates the performance of word sense disambiguation. The third set of experiments shows how much the word sense disambiguation contributes to the overall performance of feature level sentiment analysis. 


\section{A. Evaluation of Feature Scope Identification}

In order to measure the efficiency of the feature scope identification method, reviews about mobiles are extracted using the reviews extraction method from e-commerce and review sites. The existing feature extraction method [4] is used in order to extract mobile features about which opinions are expressed. Seven hundred reviews which had good English are selected. The proposed heuristic based method is used to identify the feature scopes from these reviews. There were 1505 sentences in these reviews where 2721 opinions about different mobile features are expressed.

The results of the proposed method are compared with clause based [6], sentence based [11], [12] and adjective based [4], [13] methods in Fig. 1. The results show that the accuracy of the proposed method is $88 \%$, which is better than existing methods. Conversely, the accuracy of the sentence based method is the worst of all these methods, i.e. the accuracy is only $32 \%$. The accuracy is worst because in most cases it was observed that opinions about multi features are expressed in a single sentence. However, the sentence based method is not able to distinguish opinion of one feature from another. On the other hand, the accuracy of the clause and adjective based methods are close to each other, i.e. 47.7 and $47.2 \%$ respectively. The accuracy of clause based method is low because it does not consider the succeeding clauses where opinion about the same feature can be expressed. On other hand, the accuracy of adjective based method is not good because it only considers adjective but other parts of speech (i.e. verb, adverb and noun) also carry opinion. An important aspect is that the feature scopes wrongly identified by the proposed and clause based methods are not completely false because at least one clause about a feature is identified instead of all the clauses.

\section{B. Evaluation of Local Context Disambiguation}

Another set of experiments is performed to evaluate the performance of local context disambiguation method. As the local context depends on the four semantic relations therefore this experiment is performed in order to evaluate that how accurately our system identifies these semantic relationships. For this purpose 2381 feature scopes are extracted from 257 reviews using the proposed feature scope identification method. There were 2128 adjective and feature/noun relationships (JJ and PF/NN), 841 verb and feature/noun relationships (VB and $\mathrm{PF} / \mathrm{NN}$ ), 541 adverb and verb relationships (RB and VB), 351 adverb and adjective relationships (RB and JJ) and 134 noun and feature relationships (NN and PF). The proposed heuristics based method is used to identify these semantic relationships.

Fig. 2 shows the accuracy of the proposed method for different semantic relationships. The results shows that the overall accuracy of semantic relationships is $87.3 \%$. The accuracy of the JJ and PF/NN semantic relationship is the best, i.e. $97.7 \%$. Similarly, the accuracy of RB and VB, VB and PF/NN, JJ and PF/NN and NN and PF are $92.3 \%, 88.2$ $\%, 83.7 \%$ and $90.2 \%$ respectively. It was observed that adverb usually appears before the part of speech with which it has semantic relationship, within the same clause. Therefore, the accuracy of semantic relationships where an adverb was involved is better than the other semantic relationships.

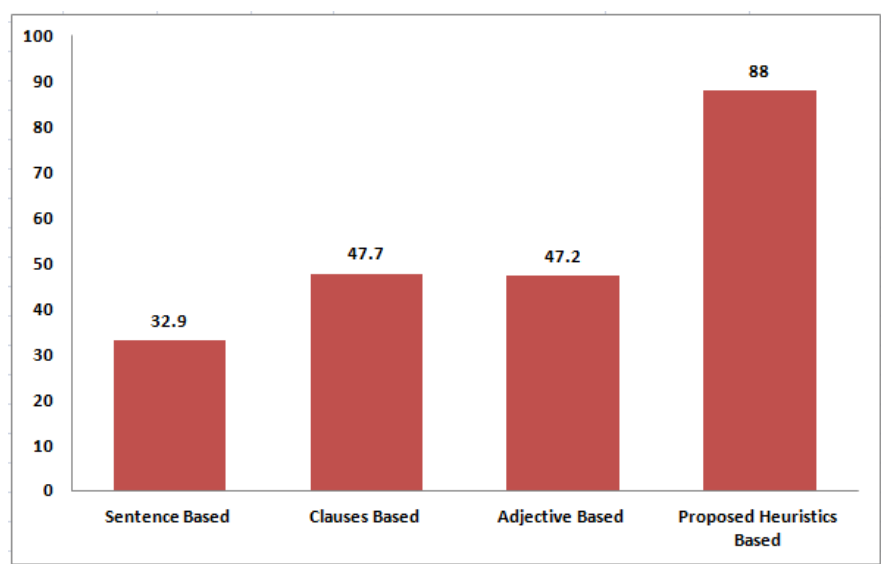

Fig. 1: Accuracy of Feature Scope Identification

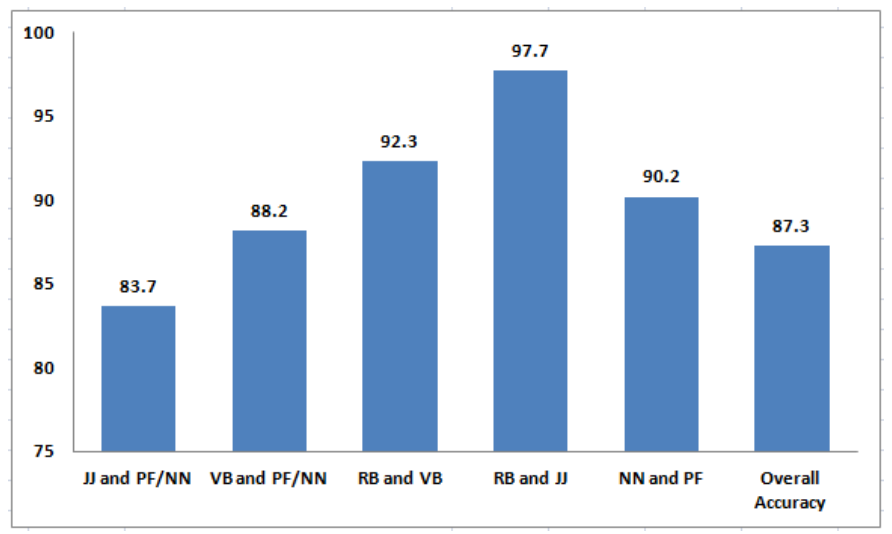

Fig. 2: Accuracy of Local Context Disambiguation

\section{Evaluation of Overall Feature Level Sentiment analysis}

In order to evaluate the performance of overall feature level sentiment analysis the mobile application domain is considered. In first step, a lexicon dictionary for mobile domain is built by using the proposed method. For this purpose 2500 reviews about mobile which have good English are extracted from e-commerce sites using the review extraction method. Product features are identified and the proposed feature scope identification method is used, which identify 3146 feature scopes in these reviews. The proposed method of building seed of word senses is used which determined 3517 seed words. All these 3517 words which include 1492 adjectives (JJ, PF), 1092 verbs (VB, PF), 460 nouns (NN, PF) and 473 adverbs (i.e. 307 (RB, JJ, PF) and 166 (RB, VB, PF)) are extracted with the corresponding contexts. The polarities are assign to the seed set by an annotator. The seed is expanded by obtaining 51848 synonyms and antonyms from WordNet, i.e. 37914 synonyms and 10417 antonyms are obtained. In this way, a lexicon dictionary which contains 55365 opinionated words with contexts and senses are built.

In the second step, the lexicon built is used in order to determine the feature level sentiment analysis. For this purpose 3000 reviews are extracted. However, these reviews are different from the reviews extracted for building lexicon dictionary. Table I shows the performance of the proposed 
TABLE I: Performance of Feature Level Sentiment Analysis

\begin{tabular}{|l|c|c|c|c|}
\hline & Positive & Negative & Neutral & Overall \\
\hline Instances & 2378 & 1250 & 186 & 3558 \\
\hline Accuracy & 94.1 & 94.2 & 98.0 & 93.2 \\
\hline Precession & 0.95 & 0.90 & 0.82 & 0.98 \\
\hline Recall & 0.94 & 0.92 & 0.77 & 0.94 \\
\hline F-measure & 0.95 & 0.91 & 0.80 & 0.96 \\
\hline
\end{tabular}

feature level sentiment analysis, which is measured in term of accuracy, precession, recall and F-measure. Accuracy is actually the percentage of total number of instances accurately classified. On the other hand, the precession $P$ for a class $C$ is defined as the total number of true positive $t p$ (i.e. the instances correctly classified as belonging to class $C$ ) divided by total number of instances classified as belonging to class $C$ (i.e. true positive and false positive $f p$, which are instances incorrectly classified as belonging to class $C$ ). Similarly, the recall for a class $C$ is the number of true positive divided by the number of instances that actually class $C$ in truth. Whereas, F-measure is the harmonic mean of precession and recall. In other words, accuracy, precession, recall and F-measure are computed by Equations 1, 2, 3 and 4 respectively.

$$
\begin{gathered}
A(C)=\frac{t p+t n}{t p+t n+f p+f n} \times 100 \\
P(C)=\frac{t p}{t p+f p} \\
R(C)=\frac{t p}{t p+f n} \\
F=2 \times \frac{P . R}{P+R}
\end{gathered}
$$

Table II compares the results of the proposed method with existing methods. The comparison is made with feature level sentiment analysis [12], [22], sentence level sentiment analysis [10] and phrase level sentiment analysis [9] methods. All these existing methods either used a word sense disambiguation method or incorporated some features to determine contextual polarity. The results show that the proposed method improved the performance of feature level sentiment analysis considerably, hence outperform the existing methods based on all evaluation measures. The accuracy of the proposed method is very good, i.e. $93.2 \%$. In addition, it also offered the best recall and precision. The significant improvement in performance is because of three main reasons. First, a lexicon dictionary is used which contains most of the words senses for a particular domain. Second, an appropriate word sense disambiguation method is adopted. Finally, all the opinionated parts of speech (i.e. adjective, verb, adverb and noun) are considered while determining the polarity.

\section{CONCLUSION}

Feature level sentiment analysis is very important for customers and manufacturers to make decisions. The two issues and causes behind errors in feature level sentiment analysis
TABLE II: Comparison of Feature Level Sentiment Analysis with Existing Methods

\begin{tabular}{|l|c|c|c|c|}
\hline \multirow{2}{*}{ Methods } & \multicolumn{4}{|c|}{ Evaluation Measures } \\
\cline { 2 - 5 } & Precision & Recall & F-measure & Accuracy \\
\hline Supervised Method [22] & - & - & - & 82.7 \\
\hline Semi-Supervised [22] & - & - & - & 64.4 \\
\hline Hu and Liu SL [12] & 0.69 & 0.64 & 0.66 & 84.2 \\
\hline \multirow{2}{*}{ Phrase Level [9] } & 0.63 & 0.67 & 0.65 & 65.7 \\
& 0.72 & 0.82 & 0.77 & 86.6 \\
WSD POS Pattern & - & - & - & 93.2 \\
Matching [10] & & & & \multirow{2}{*}{} \\
\hline Proposed Method & 0.98 & 0.94 & 0.96 & \\
\hline
\end{tabular}

are: improper sense disambiguation and inability to locate the text where opinion about a feature is expressed. In order to address these issues, in this paper we proposed a feature level sentiment analysis method which determines the opinions about different product features. A word sense disambiguation method is proposed which performed the following sub tasks in order to accurately disambiguate the sense of word while determining the polarity. a) Determines the exact boundaries of the text where opinion about a feature is expressed. b) Uses an appropriate method to identify the context of word in a sentence. c) Provides a context matching mechanism in order to obtain the polarity of the corresponding context from the lexicon. d) Builds a lexicon dictionary which not only contains the senses of words in a particular domain but also support a context matching mechanism. The results shows that the proposed methods considerably improved the overall performance of feature level sentiment analysis.

As future works, an argument mining method will be proposed to further analyzed the results of feature level sentiment analysis in order to determine the reasons behind customer preferences, likes and dislikes. This will allows the manufactures to make decisions more effectively. Furthermore, feature level sentiment analysis system will be integrated with PLM (Product Life Cycle Management System) in order to allow the organizations to make decisions throughout product life cycle.

\section{ACKNOWLEDGMENT}

This project has been funded with support from the European Commission (cLink Project: 372242-1-2012-1-UK-ERA MUNDUS-EMA21). This publication reflects the views only of the authors, and the Commission cannot be held responsible for any use which may be made of the information contained therein.

\section{REFERENCES}

[1] S. R. Das and M. Y. Chen, "Yahoo! for amazon: Sentiment extraction from small talk on the web," Management Science, vol. 53, no. 9, pp. 1375-1388, 2001.

[2] R. M. Tong, "An operational system for detecting and tracking opinions in on-line discussion," in Working Notes of the ACM SIGIR 2001 Workshop on Operational Text Classification, vol. 1, 2001, p. 6.

[3] K. Dave, S. Lawrence, and D. M. Pennock, "Mining the peanut gallery: Opinion extraction and semantic classification of product reviews," in Proceedings of the 12th international conference on World Wide Web. ACM, 2003, pp. 519-528. 
[4] M. Hu and B. Liu, "Mining opinion features in customer reviews," in $A A A I$, vol. 4, 2004, pp. 755-760.

[5] — "Mining and summarizing customer reviews," in Proceedings of the tenth ACM SIGKDD international conference on Knowledge discovery and data mining. ACM, 2004, pp. 168-177.

[6] A.-M. Popescu and O. Etzioni, "Extracting product features and opinions from reviews," in Natural language processing and text mining. Springer, 2007, pp. 9-28.

[7] H. Shi, G. Zhou, and P. Qian, "An attribute-based sentiment analysis system," Information Technology Journal, vol. 9, no. 8, pp. 1607-1614, 2010.

[8] C. Akkaya, J. Wiebe, and R. Mihalcea, "Subjectivity word sense disambiguation," in Proceedings of the 2009 Conference on Empirical Methods in Natural Language Processing: Volume 1-Volume 1. Association for Computational Linguistics, 2009, pp. 190-199.

[9] T. Wilson, J. Wiebe, and P. Hoffmann, "Recognizing contextual polarity in phrase-level sentiment analysis," in Proceedings of the conference on human language technology and empirical methods in natural language processing. Association for Computational Linguistics, 2005, pp. 347354.

[10] A. Khan, B. Baharudin, and K. Khan, "Sentiment classification using sentence-level lexical based semantic orientation of online reviews," Trends in Applied Sciences Research, vol. 6, pp. 1141-1157, 2011.

[11] S. Poria, A. Gelbukh, B. Agarwal, E. Cambria, and N. Howard, "Sentic demo: A hybrid concept-level aspect-based sentiment analysis toolkit," ESWC 2014, 2014.

[12] M. Hu and B. Liu, "Mining and summarizing customer reviews," in Proceedings of the tenth ACM SIGKDD international conference on Knowledge discovery and data mining. ACM, 2004, pp. 168-177.

[13] H. Zhang, Z. Yu, M. Xu, and Y. Shi, "Feature-level sentiment analysis for chinese product reviews," in Computer Research and Development (ICCRD), 2011 3rd International Conference on, vol. 2. IEEE, 2011, pp. 135-140.

[14] S.-M. Kim and E. Hovy, "Determining the sentiment of opinions," in Proceedings of the 20th international conference on Computational Linguistics. Association for Computational Linguistics, 2004, p. 1367.

[15] H. Yu and V. Hatzivassiloglou, "Towards answering opinion questions: Separating facts from opinions and identifying the polarity of opinion sentences," in Proceedings of the 2003 conference on Empirical methods in natural language processing. Association for Computational Linguistics, 2003, pp. 129-136.

[16] G. Grefenstette, Y. Qu, J. G. Shanahan, and D. A. Evans, "Coupling niche browsers and affect analysis for an opinion mining application." in RIAO, 2004, pp. 186-194.

[17] J. C. de Albornoz, L. Plaza, and P. Gervás, "Sentisense: An easily scalable concept-based affective lexicon for sentiment analysis." in LREC, 2012, pp. 3562-3567.

[18] S. Baccianella, A. Esuli, and F. Sebastiani, "Sentiwordnet 3.0: An enhanced lexical resource for sentiment analysis and opinion mining." in $L R E C$, vol. 10, 2010, pp. 2200-2204.

[19] S. Cerini, V. Compagnoni, A. Demontis, M. Formentelli, and G. Gandini, "Micro-wnop: A gold standard for the evaluation of automatically compiled lexical resources for opinion mining," Language resources and linguistic theory: Typology, second language acquisition, English linguistics, pp. 200-210, 2007.

[20] C. Strapparava, A. Valitutti et al., "Wordnet affect: an affective extension of wordnet." in LREC, vol. 4, 2004, pp. 1083-1086.

[21] H. T. Ng, "Getting serious about word sense disambiguation," in Proceedings of the ACL SIGLEX Workshop on Tagging Text with Lexical Semantics: Why, What, and How, 1997, pp. 1-7.

[22] B. Yang and C. Cardie, "Context-aware learning for sentence-level sentiment analysis with posterior regularization," in Proceedings of ACL, 2014. 